\title{
Influence of traps in gate oxide-Si film transition layers on FD MOSFET's characteristics at cryogenic temperatures
}

\author{
V.S. Lysenko*, I.P. Tyagulsky, I.N. Osiyuk, A.N. Nazarov \\ V. Lashkaryov Institute of Semiconductor Physics, NAS of Ukraine, 03028 Kyiv, Ukraine \\ *Corresponding author: phone/fax +38(044)5256177; e-mail: lysenko@lab15.kiev.ua
}

\begin{abstract}
The results of experiments on the influence of recharging the electron traps in a $\mathrm{Si}-\mathrm{SiO}_{2}$ transition layer on the low-temperature characteristics of fully depleted silicon in insulator MOSFET devices are presented. It is shown that the low-dose gammaradiation improves electrophysical parameters of the transition layer.
\end{abstract}

Keywords: GaAs field-effect transistor, Schottky barrier, CVC.

Manuscript received 15.03.07; accepted for publication 24.04.07; published online 19.10.07.

\section{Introduction}

Creation of submicron MOS devices and integrated circuits with employing the SOI technologies is one of the main directions of development of low-power hightemperature electronics. Nanoscaling of MOS devices demands to decrease the gate oxide thickness to $2-3 \mathrm{~nm}$ for standard thermal $\mathrm{SiO}_{2}$. Such ultra-thin dielectric layer is transparent for a tunnel current, which can stimulate undesirable effects in the kinetics of transition processes during the adjustment of working regimes in transistors. In the case of thermal $\mathrm{SiO}_{2}$, another limiting factor in nanoscale MOS devices is the transition layers in the $\mathrm{Si}_{-} \mathrm{SiO}_{2}$ interface of about $1 \mathrm{~nm}$ in thickness. In the transition layer, the oxide structure properties are different as compared with the bulk oxide. The transition layers with distorted angles of Si-O-Si bonds (which correlates with the value of the mechanical compression stress near the $\mathrm{Si}_{-} \mathrm{SiO}_{2}$ interface) were observed even in extremely uniform ultra-thin oxide [1].

In our previous papers, we studied the spectra of electron traps in $\mathrm{Si} / \mathrm{SiO}_{2}$ transition layers of the MOS structures using the thermally stimulated charge release and dynamic current-voltage characteristics methods in the temperature range 6-30 K. In [2-6], it was shown that

1) two systems of transition layer centers exist near the conduction (shallow electron traps) and valence (shallow hole traps) band edges of silicon, and these centers are separated from Si by a potential barrier;

2) the exchange of carriers between these centers and the corresponding allowed bands are determined by the tunnel-activation or tunnel mechanisms;

3 ) the activation energy of the carriers released from these centers is found to be ranged from 10 to
$70 \mathrm{meV}$ that is determined most likely by phonons associated with defects in the transition layer;

4) the processes of carrier emission from these centers to the deep depletion region of $\mathrm{Si}$ were observed at temperatures from 6 to $20 \mathrm{~K}$.

In this work, we show that the recharging of these centers can influence the low-temperature characteristics of fully depleted (FD) silicon-on-insulator (SOI) MOSFET devices at temperatures below $20 \mathrm{~K}$. It have been demonstrated that low-dose gamma-radiation improves electronic properties of the transition layer.

\section{Experiment and results}

Inversion Mode (IM) $n$-channel MOSFETs fabricated at the Louvain La Neuve University on the base of UNIBOND SOI wafers are studied. The BOX, Si film, and gate oxide thicknesses were 360,80 , and $38 \mathrm{~nm}$, respectively. Channel doping was $N_{A}=5 \times 10^{16} \mathrm{~cm}^{-3}$. The width was $172 \mu \mathrm{m}$, and the length was $3 \mu \mathrm{m}$. $\mathrm{A} \mathrm{Co}^{60}$ source supplied the gamma-radiation with doses of $10^{3} \ldots 10^{4}$ rad. MOSFETs were shorted during irradiation time.

The charging of hole traps in the Si film-gate oxide interface was performed by application of a negative bias $V=-2 \mathrm{~V}$ to the gate at a temperature of $30 \mathrm{~K}$, which resulted in the accumulation of holes in the interface of a silicon film. The drain, source, and substrate of the transistor were grounded during charging. Then the transistor was cooled to a lower temperature, the charging bias was turned off, and working biases were applied to its terminals. The input and output transistor characteristics were measured prior to and after the filling of traps with a voltage sweep rate 
of $0.05 \mathrm{~V} / \mathrm{s}$. In almost all measurements, the low drain bias was used (from 50 to $100 \mathrm{mV}$ ) to avoid the impact ionization, avalanche breakdown, and hot carrier effects. Characteristics of the devices were measured using a grounded body contact to decrease the body potential floating and interface coupling effects. The transient behavior of transistors with charge interface traps was studied also by measuring the time evolution of the drain current transient at different temperatures (isothermal regime) and by measuring the temperature dependences of the drain current during a linear heating (thermostimulation regime) of transistors.

In Fig. 1, we present the input characteristics of FD transistors measured at $10 \mathrm{~K}$ for the case of initially empty and initially filled traps in the transition layer of the gate oxide-Si film interface. One can see that the filling of traps followed by the negative shift of characteristics indicating a decrease of the front channel threshold voltage is related to the capture of a positive charge in the Si film-gate oxide interface. From the shift of the threshold voltage (Fig. 1, curves "init" and 1), the density of the positive charge was found to be (4-5) $\cdot 10^{10} \mathrm{~cm}^{-2}$. The quasi-parallel shift of the drain current vs the gate voltage with small hysteresis (the difference between the forward and reverse runs of curves) testifies that the relaxation of a trapped charge is small in the region of the uniform inversion channel. The differences in the value of relaxed charge between the first and second, the second and third voltage sweeps on the gate was calculated to be $1.8 \cdot 10^{10}$ and $0.4 \cdot 10^{10} \mathrm{~cm}^{-2}$, respectively. For the recovery of the initial characteristics, it is necessary to carry out several sweeps.

Influence of low-dose gamma irradiation on the low temperature input characteristics is shown in Fig. 2. Small increase of the threshold voltage in devices is observed after irradiation with a dose of $5 \cdot 10^{3} \mathrm{rad}$. This indicates the building up of a negative charge in the front oxide in the case of initially empty traps [7]. The charging of traps during the cooling of irradiated

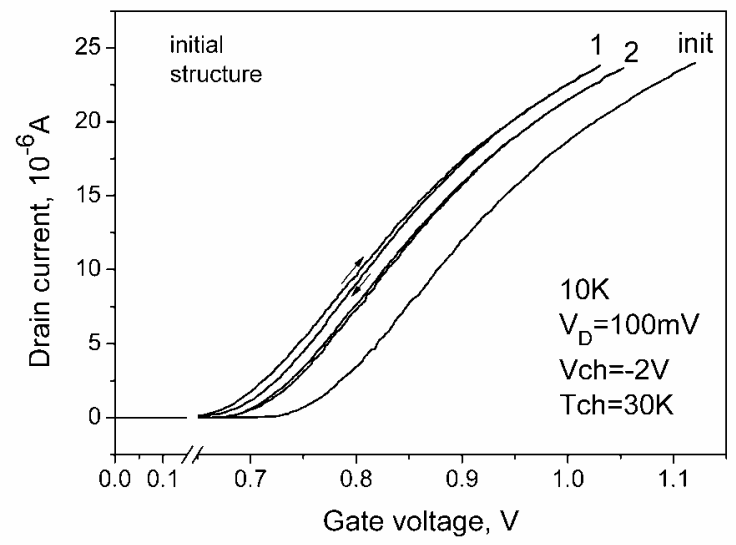

Fig. 1. Input characteristics after the traps filling of an n-channel FD SOI transistor for serial measured cycles. transistors with an applied gate bias demonstrates a negative shift of the input characteristics due to the capture of a positive charge in the front oxide similarly to non-irradiated devices. But the density of traps capturing a positive charge decreases to $8 \cdot 10^{9} \mathrm{~cm}^{-2}$ after gamma irradiation.

The output characteristics measured at $10 \mathrm{~K}$ in the inversion mode and in the subthreshold region for empty and filled interface traps are presented in Fig. 3a, b. The charging of hole traps results in an essential increase of the current in the initial part of the curve corresponding to the capture of a positive charge in oxide. After reaching the certain value of drain bias, corresponding to the beginning of the non-linear mode of transistor operation $\left(V_{\text {D.sat. }}\right)$, the curve gradually approaches the respective curves for empty traps. During the sweeping in the subthreshold region, the relaxation of the positive charge occurs faster than that in the case of the inversion mode.

Since the extra current seems to be related to the charged state of hole traps, we have studied the isothermal transient current if the transistor is switched on after the preliminary filling of the traps. Such measurements allowed us to study the kinetics of charge release depending on the temperature and the transistor operation mode. In this case, the transistor was turned on after filling the hole traps, and the drain current was measured as a function of time at a fixed temperature. Transient currents were measured in two modes. The first one corresponds to the linear mode of transistor operation $\left(U_{\mathrm{D}}=0.1 \mathrm{~V}\right)$, and the second one does to the nonlinear mode $\left(U_{\mathrm{D}}>0.2 \mathrm{~V}\right)$.

Transient behavior of the transistor is shown in Fig. 4. It is seen that, in the ohmic mode, the isothermal transient current can be fitted by an exponential relaxation for almost all of the curves. At temperatures below $14 \mathrm{~K}$, the current changes slowly for a long time. The Arrhenius plot of the time constant $\tau$ is a straight line in the temperature range $14-19 \mathrm{~K}$, that allows the

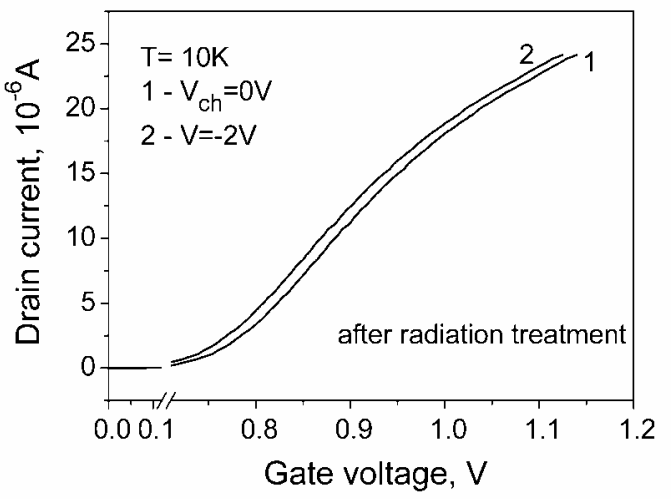

Fig. 2. Input characteristics of an irradiated transistor for empty (1) and filled (2) traps. 
calculation of the activation energy of charge release using the equation $\tau=\tau_{0} \exp \left(-\frac{E_{a}}{k T}\right)$. The activation energy of the relaxation processes derived from this curve is of the order of $13 \mathrm{meV}$. For the non-linear mode, the output characteristic transient time becomes much shorter, and the relaxation process cannot be fitted by the exponential law.
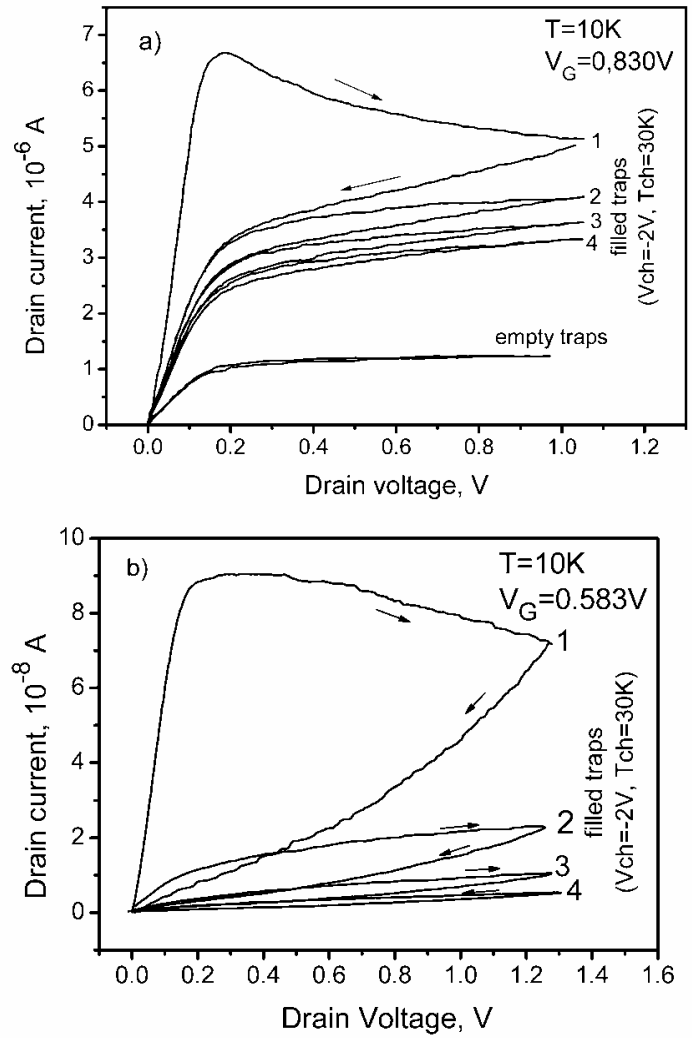

Fig. 3. Drain characteristics after the traps filling of an $n$-channel FD SOI transistor above (a) and below (b) threshold voltage $V_{\text {th }}=0.77 \mathrm{~V}$.
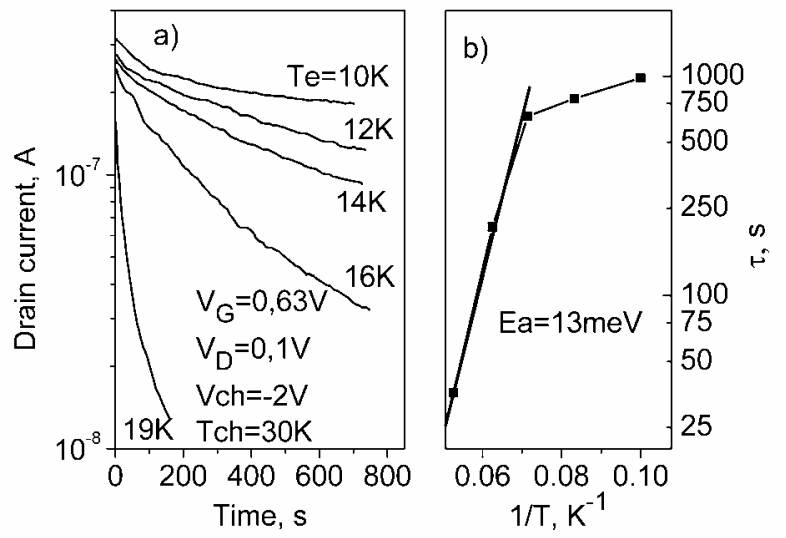

Fig. 4. Isothermal drain current relaxation at different temperatures in the linear mode (a) and the Arrhenius plot of the time constant (b) for FD SOI transistors at different temperatures.
In Fig. 5, we show the temperature dependences of the transistor drain current in the inversion mode for the cases of initially empty and initially filled interface traps. In these measurements, the filling of traps was performed by applying the gate bias $V_{G}=-2 \mathrm{~V}$ at a temperature of $30 \mathrm{~K}$. Then the sample was cooled down to $10 \mathrm{~K}$ and, after a pause of some minutes, the small fixed drain and fixed gate biases were applied to the terminals, and the current-temperature characteristics was recorded during heating. It can be seen that the positive charge of traps results in an increase of the current. When the traps are emptied (at temperatures from 15 to $30 \mathrm{~K}$ ), the current curve becomes similar to that measured without filling traps.

The temperature dependence of the drain currents at a linear temperature scan for non-irradiated and irradiated transistors after filling traps is shown in Fig. 6. It is seen that, after irradiation, the modulation of the drain current at the initial stage is decreased by a factor of 5 that is consistent with a shift of the threshold voltage of input characteristics. At the same time, the behavior of the drain current in irradiated devices became similar to that in non-irradiated samples as the traps are emptied.

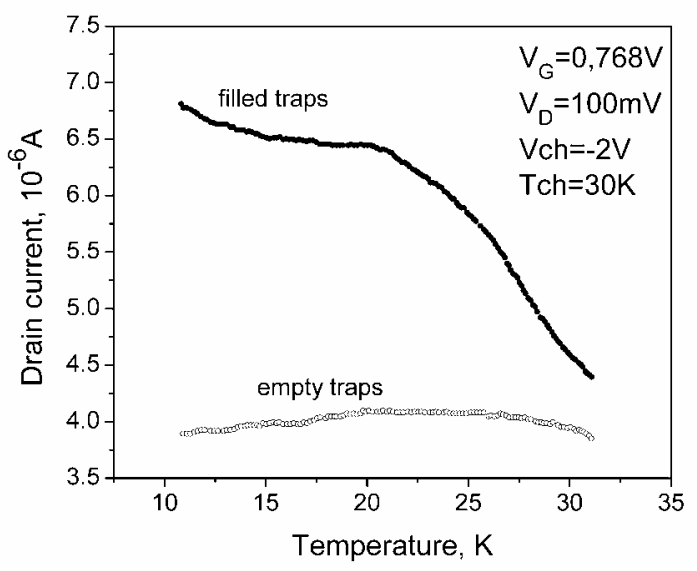

Fig. 5. Temperature dependences of the drain current for FD SOI transistors.

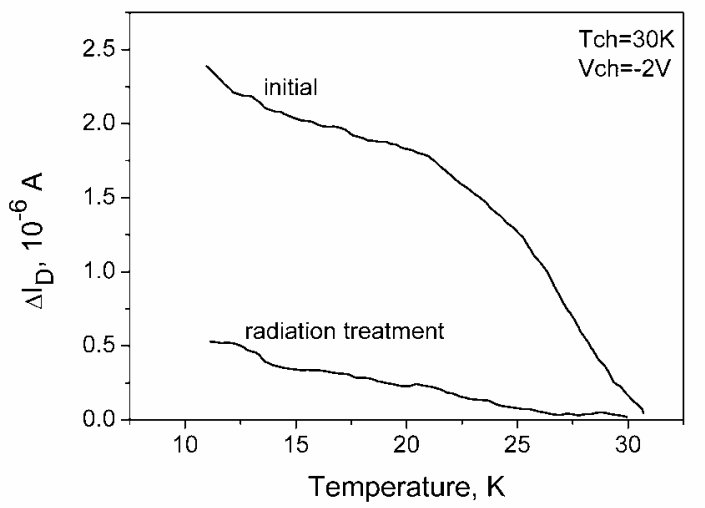

Fig. 6. Temperature dependence of the drain current of an $n$-channel FD MOSFET for filled traps before (1) and after (2) irradiation. 

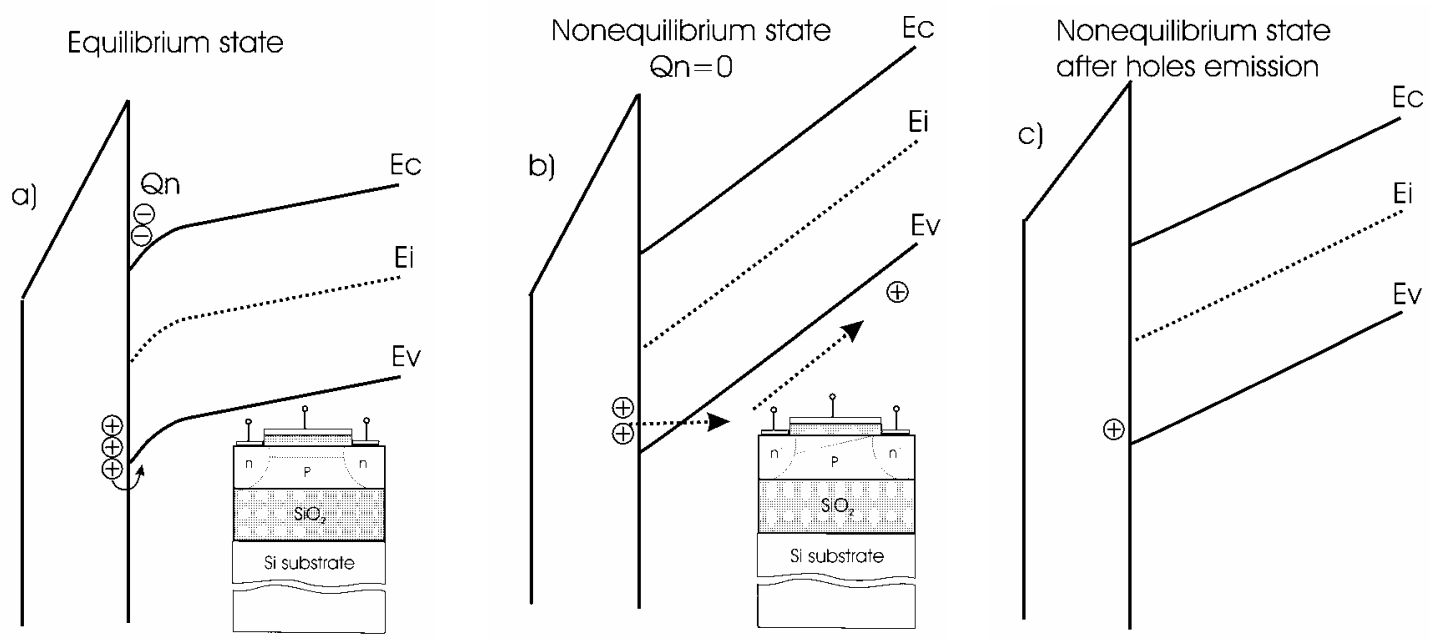

Fig. 7. Band diagram in the inversion mode. (a) - linear mode, small drain voltage, thermoactivated emission of holes from transition layer traps; (b) - pinch-off mode, the field emission of holes from transition layer traps; (c) - pinch-off mode after the emission of holes.

\section{Discussion}

The fact that the temperature ranges of the transient current and the activation energy are close to those of the earlier studied spectra of traps in transition layers proves that the non-equilibrium effects of transistor operation at low temperatures are related to the charge capturerelease on the traps located in the oxide/semiconductor transition region under the gate.

The type of input and output characteristics under conditions of our experiment allows us to conclude that charge traps in the transition layer are unstable. The trapped charge induces the corresponding shift of the threshold voltage which is a function of time during emptying traps.

Zone diagrams of the corresponding linear and non-linear modes are presented in Fig. 7a, b. When the charging process has terminated, the holes are located on traps near the silicon valence zone in the interface $\mathrm{Si} / \mathrm{SiO}_{2}$ and the transition layer. In the inversion mode, the charge associated with holes trapped by surface states on the $\mathrm{Si} / \mathrm{SiO}_{2}$ interface are recharged completely over the whole surface under the gate. At the same time, the traps located in silicon oxide are separated from silicon by a potential barrier. These traps at temperatures below $10 \mathrm{~K}$ and under the electroneutrality condition are able to retain the trapped charge for a long time (Fig. 7a).

In the linear mode of the transistor, the quasiequilibrium state of the total surface charge is established when the sum of the gate charge $\left(Q_{\mathrm{M}}\right)$, inversion layer charge $\left(Q_{n}\right)$, oxide charge $\left(Q_{o x}\right)$, and charge of ionized dopants $\left(Q_{a}\right)$ in a silicon film under the gate is about zero. In this case, the field on the drain (very small bias) and the gate does not change the potential in the silicon film. If the electric field does not penetrate into the film, then the charge captured on traps is released very slowly at low temperatures (less than $15 \mathrm{~K}$ ) and almost unchanged during the time of the measurement that leads to a slow recovery of the initial threshold voltage. At temperatures above $15 \mathrm{~K}$, the charge released in the linear mode increases, and the relaxation process is determined by the activation mechanism. The hysteresis of the current amplitude decreases.

In the case of low-dose irradiated transistors, the similar effects are observed. However, irradiation leads to a reduction of the observed effects during isothermal and thermo-stimulated measurements of drain currents. It seems that this effect is related to a decrease of the density of the traps of holes in the transition layer due to ordering the transition layer of oxide.

It was mentioned above that the positive charge captured in traps of the transition layer reduces the threshold voltage, which causes an increase of the drain current. At a sufficiently high drain voltage, when $V_{\mathrm{D}}$ reaches the pinch-off point $\left(V_{\mathrm{D}, \text { sat. }}\right)$, the transistor turns into the nonlinear mode. In the pinch-off point, the charge of the inversion layer $Q_{n}$ drops to zero (Fig. 7b). At low temperatures in fully depleted silicon films, this leads to a significant increase of the depleting surface potential. Fully depleted silicon films can be considered as insulator at low temperatures. In this case, the nonequilibrium conditions are fulfilled near the drain and along the channel. The electrical field of the gate penetrates into silicon films and assists in increasing the depleting surface potential. Influence of the field on the change of a charge in traps in the oxide transition layer is a relatively low process, in contrast to the inversion charge which responds very quickly to any changes of the potential. Considerable electric field facilitates the charge emission of carriers from hole traps in oxide to the silicon film along channels by the tunneling process. 

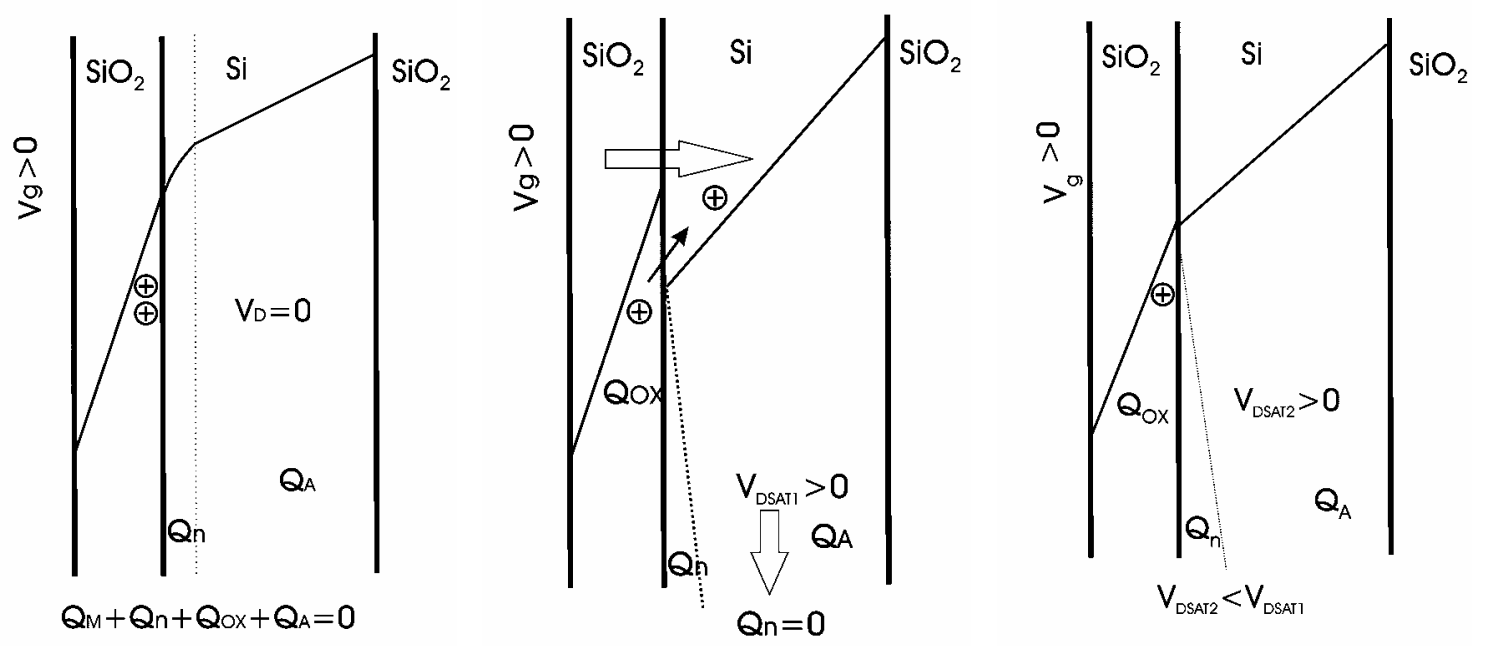

Fig. 8. Space distribution of the electrical potential of a FD SOI transistor under condition of the field emission of carriers from oxide traps.

Then holes are removed by the grounded body contact and recombined near the directly biased source. Decrease of the positive charge in oxide leads to the redistribution of electrical fields between oxide and the film that causes a rapid recovery of the threshold voltage of the transistor with time in comparison with that in the linear regime. As a sequence, the drain current is relaxed in the saturation region.

The reduced field in the dielectric and the depletion potential (in comparison with the initial state) are settled in the film after completing the field emission of carriers. If the fraction of the charge associated with captured holes remains in oxide, then the field emission from these centers will occur at a less drain voltage at the pinch-off point providing the unchanged value of drain voltage sweep and gate voltage. In the subthreshold mode, the field emission of captured holes from traps in the transition layer will be more intense because the inversion charge is small, and it leads at the same drain voltages to a higher depletion surface potential in the fully depleted film.

In Fig. 8, one can see the space distribution of electrical potential in a fully depleted SOI MOSFET under condition of the field emission of carriers from transition layer centers. The fast respond of the inversion charge to the drain voltage leads to a significant increase of the depletion potential in the film $\left(Q_{N} \rightarrow 0\right)$, and the gate field penetrates into the film. When the electrical field in the film reaches the critical value, the charge captured by traps in the oxide transition layer starts to release. The field in the insulator falls down, and a new potential distribution is settled.

\section{Conclusions}

1. The effects of instability of the FD SOI MOSFET characteristics in the region of cryogenic temperatures (10-25 K) are related to the recharging of traps in the oxide transition layer by the tunnelactivation mechanism.

2. Low-dose irradiation improves the stability of the FD SOI MOSFET characteristics due to the ordering of the transition layers $\mathrm{Si} / \mathrm{SiO}_{2}$.

3. The estimation of changes of the threshold voltages at the low-temperature charging of traps in the transition layer is the effective method for the determination of the gate oxide - silicon interface quality in SOI transistors.

Tunnel mechanisms of electrical transport involving centers in the transition layer can be useful for explanation of some effects in short-channel fully depleted MOSFETs with ultrathin gate oxide (for example, the induced kink effect and noise spectra in the linear mode).

\section{References}

1. K. Hirose, H. Nobira, T. Koike, S. Sakano and T. Hattory, Structural transition layer at $\mathrm{SiO}_{2} / \mathrm{Si}$ interface // Phys. Rev. B 59 (8), p. 5617-5621 (1999).

2. V.S. Lysenko, T.N. Sytenko, V.I. Zimenko, and O.V. Snitko, Investigation of traps in the transition region of $\mathrm{Si}_{-} \mathrm{SiO}_{2}$ structures at cryogenic temperature // Phys. status solidi (a) p. 619-625 (1982).

3. V.S. Lysenko, T.N. Sytenko, O.V. Snitko, V.I. Zimenko, A.N. Nazarov, I.N. Osyuk, T.E. Rudenko, and I.P. Tyagulskii, Interrelation between surface states and transition layer defects in $\mathrm{Si}_{-} \mathrm{SiO}_{2}$ structures // Solid State Communs 57 (3), p.171-174 (1986)

4. Yu.V. Gomenyiuk, R.N. Litovski, V.S. Lysenko, I.N. Osiyuk, and I.P. Tyagulski, Thermally stimulated field emission of charge from traps in the transition layer of $\mathrm{Si}-\mathrm{SiO}_{2}$ structures // Appl. Surf. Sci. 55 (1), p.179-185 (1992). 
5. Yu.V. Gomenyuk, R.N. Litovski, V.S. Lysenko, I.N. Osiyuk, and I.P. Tyagulski, Current stoichiometry of field emission of charge from traps in the transition layer of implanted MIS structures // Appl. Surf. Sci. 59 (1), p. 91-94 (1992).

6. V.S. Lysenko, I.P. Tyagulski, A.N. Nazarov, I.N. Osiyuk, Yu.V. Gomenyuk, Research of thermally stimulated field emission in SIMOX structures at cryogenic temperatures // Optoelektro- nika i Poluprovodnikovaya Tekhnika 40, p. 88-95 (2005) (in Russian).

7. A. Evtukh, A. Kizjak, V. Litovchenko, C. Clays, and Simoen, Radiation characteristics of short $p$ channel MOSFET's of SOI substrates // Proc. "Science and technology of semiconductors on insulators structures and devices operating in a harsh environment". Kluwer, Dordrecht, 2005, 185, p. 221-226. 